\title{
Coronary heart disease rates within a small urban area in Belgium
}

\author{
G de Backer, G Thys, I de Craene, Y Verhasselt, $S$ de Henauw
}

\begin{abstract}
Study objective - To identify geographical differences in coronary heart disease (CHD) attack rates in a small urban area and to relate these to indicators of socioeconomic class.

Design - CHD attack rates were calculated from data of the Ghent MONICA myocardial infarct register for the period 1983-87. The city of Ghent is subdivided into 201 sectors based on morphological, and socioeconomic characteristics. During the national census of 1981, the main determinants of residential differentiation were measured. These sector variables were linked with the CHD attack rates.

Patients - All residents of the city aged 25-69 years are prospectively followed with regard to heart attacks. Between 1982 and 1987, 1728 suffered an acute heart attack according to MONICA criteria.

Main results - Significant $(p<0.05)$ differences in age and sex standarised attack rates were observed between city sectors. These differences were related to an index of socioeconomic status.

Conclusion - Within a small urban area, significant geographical differences occur in CHD attack rates and these are related to socioeconomic status.
\end{abstract}

\section{( $\mathcal{E}$ Epidemiol Community Health 1994;48:344-347)}

Research into the causes of atherothrombotic coronary heart diseases (CHD) has identified numerous risk factors. ${ }^{1-5}$ Results from clinical, experimental, and epidemiological studies indicate that the pathogenesis and the occurrence of clinical events are multifactorial in origin. Risk factors relate to environmental differences, life styles, and genetic phenotypes, and between all these exist interactions that may be additive or synergistic.

Differences in CHD occurrence between study populations can largely be explained by differences in the major risk factors such as arterial blood pressure, smoking habits, and some blood lipoprotein levels. ${ }^{6-7}$ Differences in incidence within populations, however, are incompletely explained. ${ }^{8}$ Smoking, arterial hypertension, and hyperlipidaemia may account for half of all new cases and research with an emphasis on pyschosocial, genetic, and other environmental variables continues.

A more recent epidemiological development for studying the causes of disease is the examination of localised clustering of disease. Within the field of cancer epidemiology, in particular, several groups are now working to develop a better small area data base and to improve analysis systems for studying geographically localised clustering. ${ }^{910} \mathrm{We}$ have tried, in a similar way, to identify the spatial pattern of CHD in an urban area subdivided into small homogenous areas with the idea of analysing possible relationships with variables available at that geographical level.

We were particularly interested in any possible associations between disease and socioeconomical factors. In Belgium the reliability of national mortality statistics according to socioeconomic class differences is very poor. In the Belgian Heart Disease Prevention Project of the 1970s, a " $U$ " shaped relationship between the professional class and CHD incidence was observed with the highest incidence in blue collar workers and executives and an intermediate value in white collar workers. ${ }^{11}$ It could be that at that time the population was in transition between what was originally called a "manager's disease" (occurring more frequently in the higher social classes) and the actual situation in the northern European countries, where CHD occurs more frequently in lower socioeconomic groups. ${ }^{12-14}$

The objective of this study, therefore, was to identify geographical differences in CHD attack rates and to relate these to indicators of socioeconomic class.

\section{Methods}

In the city of Ghent, Belgium, a register for acute myocardial infarction has been in operation since 1 January 1983 within the framework of the MONICA project. The objectives and methodology of MONICA have been published previously. ${ }^{15}$ Ghent has approximately 236000 inhabitants. The target population for the register are all men and women aged 25-69 years - that is approximately 131000 subjects.

From 1983 to date a registration system based on a well defined operational definition of acute coronary events has been used. This is based on clinical symptoms, electrocardiographic criteria, cardiac enzyme results, autopsy data, and personal antecedents. Cases are entered in the register as definite or possible myocardial infarction or as cardiovascular deaths without further proof. Definitions of these categories have been published. ${ }^{15}$ Each year 300 to 400 events occur in our target population. For this study we have used all 1728 cases entered during the years $1983-87$ $48 \%$ of these were definite infarction, $33 \%$ possible infarction, and $19 \%$ cardiac deaths ascribed to an acute myocardial infarction. 
Between 1983 and 1987, 80 of the 1728 subjects were entered the register more than once. In approximately $80 \%$ of all the cases, the episode that led to registration was the first event and in $20 \%$ a recurrent attack. Therefore $\mathrm{CHD}$ occurrence is expressed in attack rates. Results on the completeness and on the quality of the register have been published. ${ }^{1617}$

Ghent occupies an area of $156 \mathrm{~km}^{2}$ and has a population density of 1507 inhabitants $/ \mathrm{km}^{2}$. It is subdivided into 201 statistical sectors (fig 1) ranging in area from 7 to 710 hectares, with an average of $79 \mathrm{ha}$. The average number of inhabitants per sector in 1981 was 1190 (range 0 to 5361). The sectors were defined in 1970 by the National Statistical Institute on the occasion of the 13th national census. The subdivision complied with an international recommendation to aggregate census results on a level smaller than that of a community. The whole of Belgium was subdivided into statistical sectors defined as small homogenous territorial units differing from the surrounding area on morphological, functional, and social grounds. ${ }^{18}$

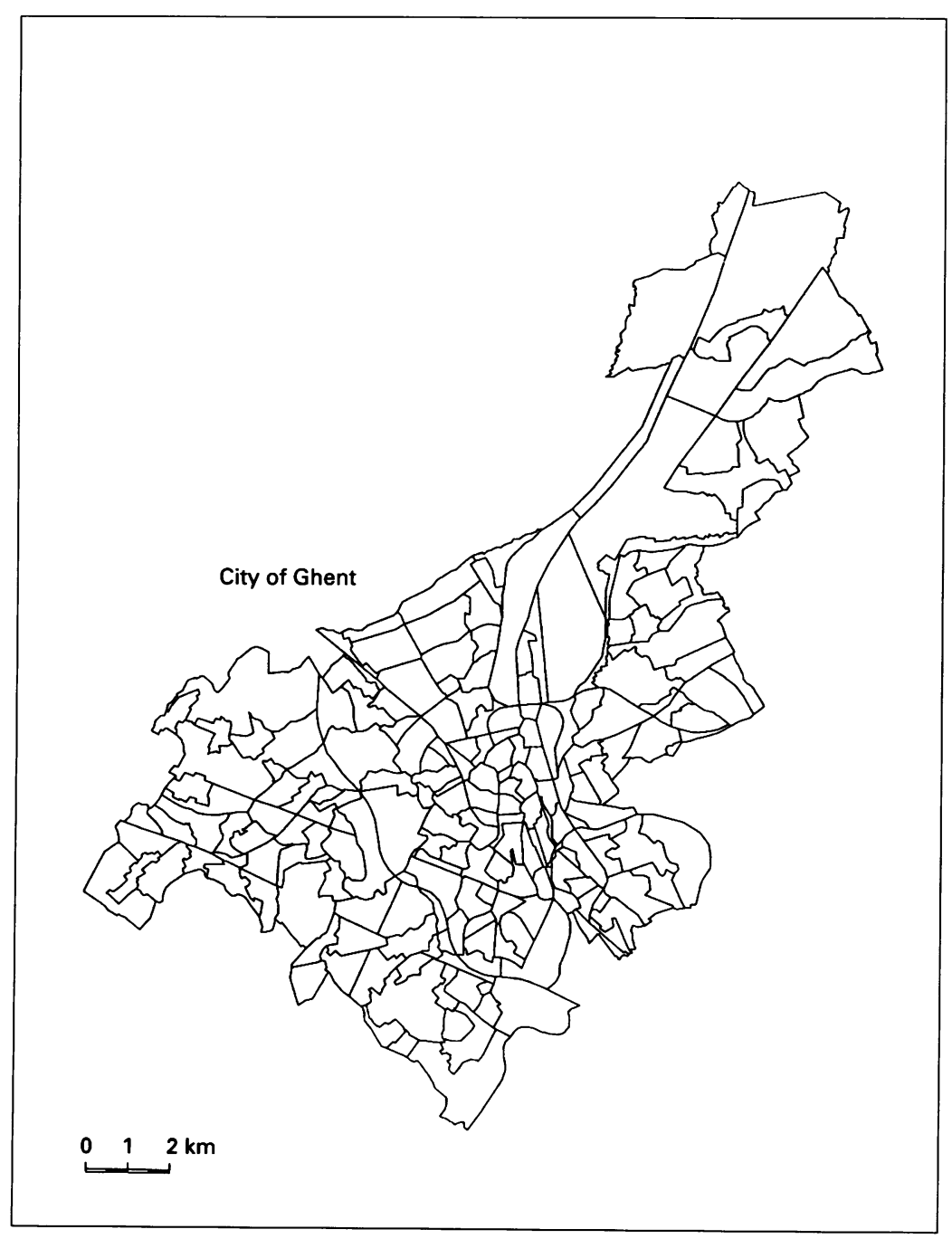

Figure 1 The city of Ghent subdivided into 201 statistical sectors (all maps were drawn by the Geography Institute, Vrije University, Brussels).
To enable analysis of the spatial distribution, each case from the registry was assigned to a sector by means of its address. From the crude attack rates, the age and sex standardised rates were then calculated for each sector. Indirect standardisation was applied in order to avoid extreme local age and sex specific attack rates. This required age and sex specific attack rate data for the standard population. As these were not available on a national scale, the population of Ghent was used as the standard population.

To compare the resulting spatial distribution with that of possible explanatory factors, a database was constructed containing 74 variables derived from the national census of 1981 . These were selected to measure as many aspects as possible of family status, socioeconomic status, and ethnic status of the population. These factors are said to be the main determinants of residential differentiation in cities. ${ }^{19}$ Submitting a selection of 25 variables (table 1) to a factor analysis revealed two factors which explained $56 \%$ of the total variance and corresponded to the definition of family status and socioeconomic status. For convenience we replaced these by the highest loading variables ( $\%$ of families with three or five members and $\%$ of executives respectively). The results obtained are very similar to those of earlier studies on residential differentiation in Belgian cities. ${ }^{20}$

A two-tailed statistical test was conducted to determine whether a particular attack rate differed significantly from the mean for Ghent. We used a 0.05 level of significance and the Poisson distribution to describe the possible outcomes. ${ }^{21}$ The same test was applied to evaluate socioeconomic or family status deviations from the mean for sectors aggregated according to the attack rate.

Table 1 Variables submitted to factor analysis

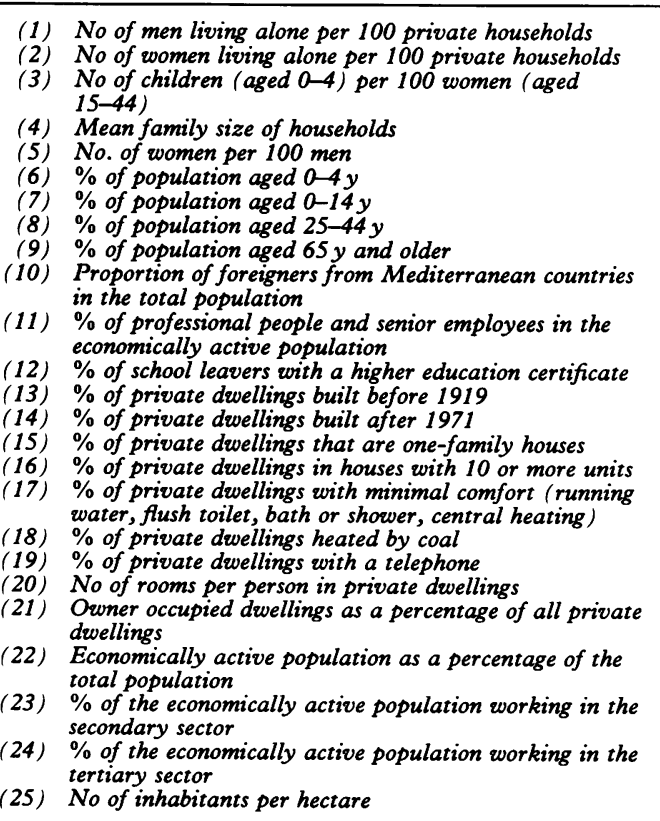




\section{Results}

Figure 2 shows the spatial distribution of the age and sex standardised CHD attack rates, excluding sectors with no inhabitants. Rates range from less than 10 to more than 50 per 10000 inhabitants per year. Figure 3 presents a map of sectors with attack rates that differ significantly from the mean. Higher attack rates are shown in black, lower rates in white. Although the spatial distribution of the attack rates does not show a clear pattern, both maps confirm that important differences in $\mathrm{CHD}$

Table 2 Socioeconomic and family status in sectors with significantly high and low coronary heart disease $(C H D)$ attack rates

\begin{tabular}{llll}
\hline & \multicolumn{2}{l}{ Sectors aggregated by CHD attack rates } \\
\cline { 2 - 4 } & & \multicolumn{2}{c}{ Significantly high/low rates } \\
\cline { 2 - 4 } & Mean & Low & High \\
\hline CHD attack rate $/ 10000$ & $26 \cdot 2$ & $4 \cdot 5$ & $48 \cdot 8$ \\
$\%$ Executives & $16 \cdot 7$ & $20 \cdot 6^{*}$ & $12 \cdot 8^{*}$ \\
$\%$ Families with 3 or 4 persons & $29 \cdot 4$ & $29 \cdot 1$ & $29 \cdot 0$ \\
\hline * Different from the mean $p<05$ & &
\end{tabular}

${ }^{*}$ Different from the mean $\mathrm{p}<.05$

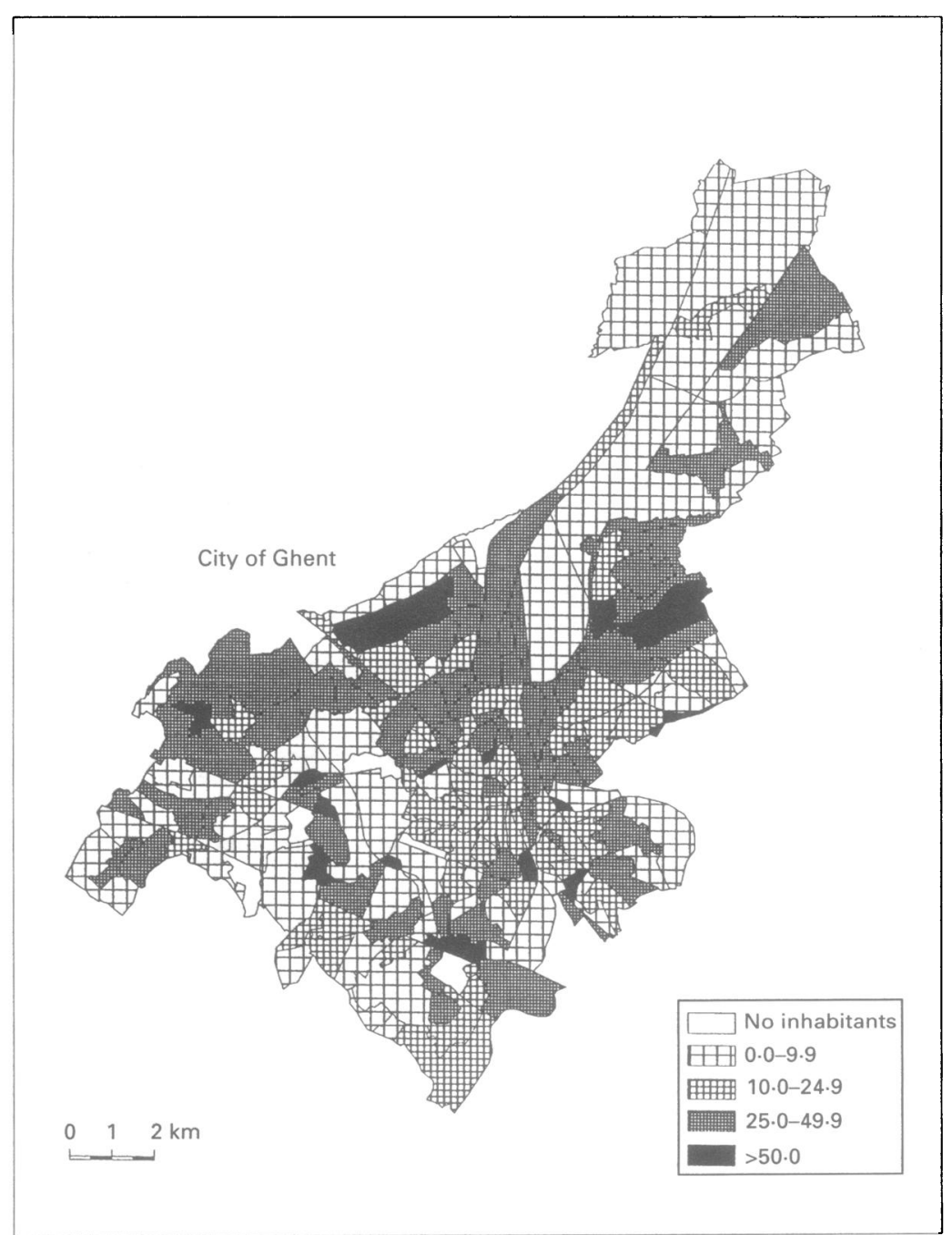

Figure 2 Age and sex standardised coronary heart disease attack rates (per 10000/y) in the statistical sectors of Ghent 1983-87. attack rates do exist between urban sectors.

The age and sex standardised CHD attack rates per sector were then related to the two factors reflecting the family status and the socioeconomic status, and to the variables that were used to construct them. For aggregations of sectors, however, a significant relation with the socioeconomic status was found (see table 2). An aggregation of the sectors with significantly low attack rates results in socioeconomic status (measured by the percentage of executives) that was significantly higher than the mean. A significantly low socioeconomic status was found for the aggregation of sectors with significantly high $\mathrm{CHD}$ attack rates. No significant association was found for the family status (measured by the proportion of families with three or four persons).

\section{Discussion}

Studies on the geographic differences in incidence and in temporal trends of CHD mortality have contributed to our understanding of the possible role of environmental factors. In the 60 s and 70 s differences between countries were largely explicable by differences in life styles. Since 1970, heterogeneous trends in CHD mortality have been observed in the USA, Canada, and Australia, while in several eastern European countries an alarming increase can be seen. The reasons for these divergent trends are unclear and are the objective of continuing research. ${ }^{15}$

Within populations, differences in CHD incidence are less well explained. Other factors related to social class, genetics, psychological traits, and geochemical factors have been suspected. Regional differences within countries have been shown, but are not fully understood. On the contrary, in studies on regional differences within a nation it is generally assumed that a regional area such as a city should be treated as a homogeneous entity. Intra-urban analysis of CHD attack rates within Ghent suggests that substantial variation exists. Differences in CHD attack rates on the local level of one particular rural or urban area have rarely been reported. In Savannah, Georgia, USA, differences in stroke incidence were reported between urban areas. ${ }^{22}$ In the neighbourhoods of Ghent, age and sex standardised CHD attack rates are almost as different as those observed between west European populations. The geographical picture is difficult to understand, however, because of the scatter over the whole area. No strong relationship was observed between differences in the attack rate and differences in socioeconomic factors reflected by the 1981 census data. The quality and reliability of this information can be questioned. This may be due to the limited statistical power of the study. Indeed small area statistics require sufficient numbers of events in small areas which is only possible by aggregation over several years of morbidity or mortality statistics, or both. At the end of the 10 years of $\mathrm{CHD}$ attack registration in Ghent we will have approximately double the number of events and this will allow us to recalculate 


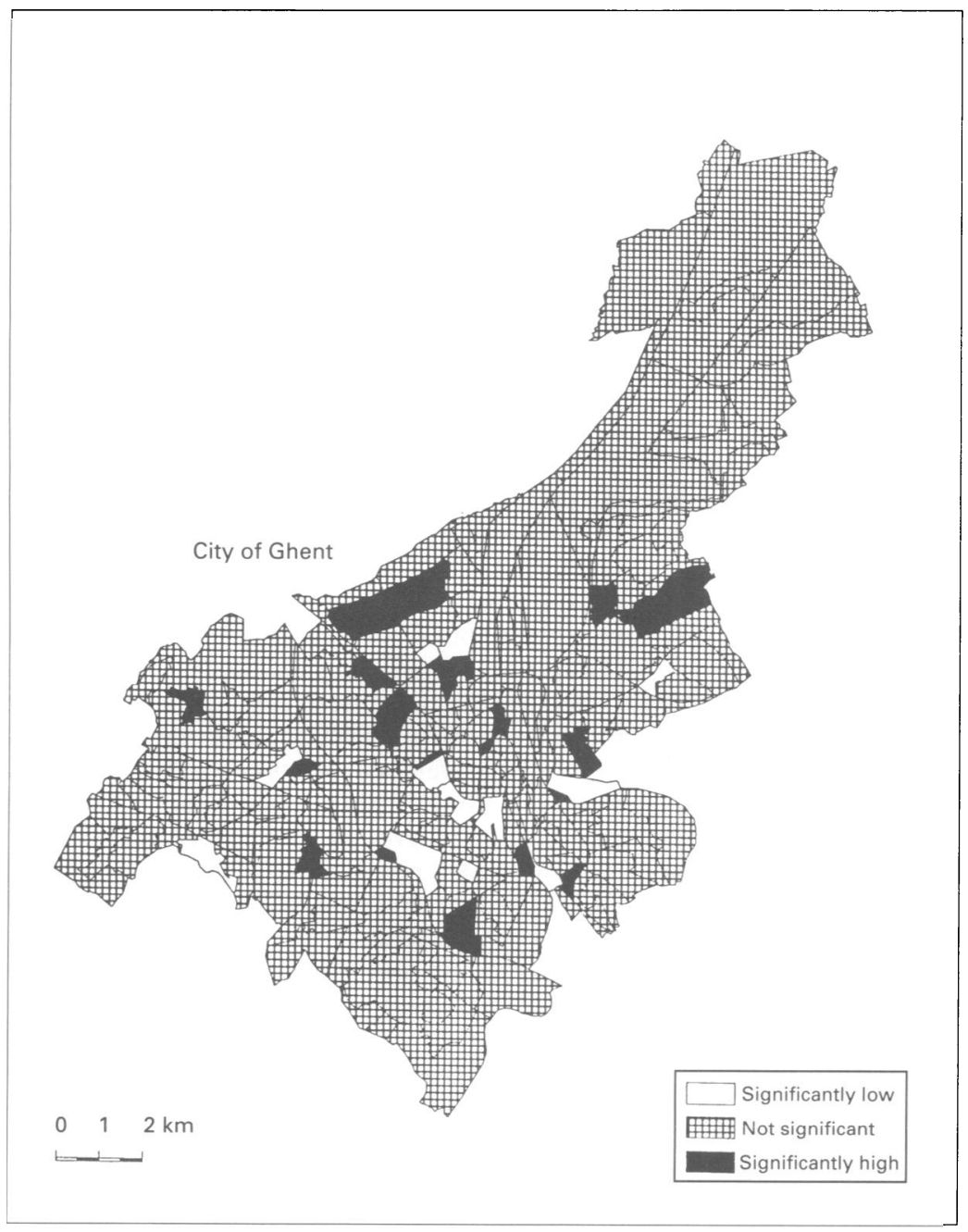

Figure 3 Statistical sectors in the city of Ghent with age and sex standardised coronary heart disease attack rates significantly $(p<0.05)$ below ( $\square$ ) or above the expected value.
These observations support the hypothesis that heart attacks are related to socioeconomic factors. The identification of these areas makes the detailed study of possible explanations worthwhile. At the micro-scale of a neighbourhood it becomes easier to control for socioeconomic factors and to determine environmental and behavioral factors more precisely. All this can be of help in the development of primary and secondary prevention CHD programmes within urban areas.

1 Martin MJ, Hulley SB, Browner W, Kuller LH, Wentworth D. Serum cholesterol, blood pressure and mortality. Implications from a cohort of $361662 \mathrm{men}$. Lancet 1986;ii:933-6.

2 Jenkins CD. Recent evidence supporting psychologic and social risk factors for coronary disease. $N$ Engl $f \mathrm{Med}$ 1976;294:987-94.

3 Paffenbarger RS, Wing AL, Hyde RT. Physical activity as an index of heart attack risk in college alumni. $A m \mathcal{F}$ an index of heart attack risk

4 Logan RL, Riemersma RA, Thomson M, et al. Risk factors for ischaemic heart disease in normal men aged 40. Edinburgh-Stockholm Study. Lancet 1978;i:949-55.

5 Cambien F, Poirier O, Lecerf L, et al. Deletion polymorphism in the gene of antiogensin-converting enzyme is a potent risk factor for myocardial infarction. Nature 1992; 359:641-4.

6 Keys A. Coronary heart disease in seven countries. Circulation 1970;41,(suppl): 1 .

7 Inter society commission for heart disease resources. Primary prevention of atherosclerotic diseases. Circulation 1970;42. prevention.

8 Marmot MG, Shipley MJ, Rose G. Inequalities in death specific explanations of a general pattern. Lancet 1984;i:1003-6.

9 Alexander FE. Methods of mapping and identifying small clusters of rare diseases with applications to geographical epidemiology. Geographical Analysis 1991;23(2):158-73.

10 Gardner MJ, Snee MP, Hall AJ, Powell CA, Downes S Terrell JD. Results of case-control study of leukemia and ymphoma among young people near Sellafield nuclear plant in West Cumbria. $B M \mathcal{F}$ 1990;300:423-9.

11 Kornitzer M, Dramaix M, Kittel F, De Backer G. Classe sociale et risque de cardiopathie ischemique. La Revue du Practicien 1981;31:3805.

12 Holme I, Helgeland A, Hjermann I, Leren P, Lund-Larsen PG. Four year mortality by socioeconomic indicators: the Oslo study. 7 Epidemiol Community Health 1980;34:48.

13 Rose G, Marmot M. Social class and coronary heart disease. Br Heart 7 1981;45:13.

14 Carstairs V, Morris R. Deprivation and health in Scotland. Aberdeen: Aberdeen University Press, 1991.

15 WHO MONICA Project Principal Investigators. The World Health Organisation MONICA project (monitoring trends and determinants in cardiovascular disease): a major international collaboration. $\mathcal{f}$ Clin Epidemiol 1988;41(2):105-14.

the attack rates in each neighbourhood with more precision. It is also recognised that the population data from which the event rates were calculated were derived from a population census that took place in 1981 and is only repeated in Belgium every 10 years. Given the demographical changes that can occur with small population groups, the extent to which small area statistics may be biased by errors in population data is unclear.

From the study of associations on a larger geographical scale a small but significant relation was observed, suggesting a higher attack rate in areas with lower socioeconomic status. 